\title{
Anticipatory responding in rats with septal lesions*
}

\author{
CHARLES F. FLAHERTY $\dagger$, LEONARD W. HAMILTON, and SALVATORE CAPOBIANCO $\dagger$ \\ Rutgers, The State University, New Brunswick, New Jersey 08903
}

\begin{abstract}
Rats were trained on a discrete-trial two-lever "counting" task that required five responses on a ratio lever and then one response on a reward lever. Rats with septal lesions were found to make more reward-lever responses prior to completion of the ratio response than control rats, and, in general, more of these anticipatory responses occurred the closer the lesioned rats were to the completion of the ratio. A shift from small to large reward reduced the number of reward-lever responses, but the anticipatory nature of these responses was still clearly evident in the lesioned rats.
\end{abstract}

Septal lesions alter the behavior of rats in a wide variety of operant tasks. For example, increased rates of responding have been found on CRF and FI schedules (e.g., Ellen \& Powell, 1962), on DRL schedules (e.g., Ellen, Wilson, \& Powell, 1964; MacDougall, Van Hoesen, \& Mitchell, 1969), and on some FR schedules (Buckland \& Schwartzbaum, 1970). Of particular relevance to this study are the effects of septal lesions on a two-lever ratio "counting" task devised by Mechner (1958a, b). This task requires a rat to complete a ratio requirement on one lever (the ratio lever) and then press once on a second lever (the reward lever). Two studies (Ellen \& Kelnhofer, 1971; Van Hoesen, MacDougall, \& Mitchell, 1972) have shown that the performance of rats with septal lesions is different from control rats on this task. In the Ellen and Kelnhofer study, operated rats were found to respond on the reward lever before the ratio requirement had been completed. Because the design of this experiment was such that early responses on the reward lever reset the ratio counter, the operated animals also showed more total response on both ratio and reward levers. Ellen and Kelnhofer (1971) attributed the performance of the operated rats to an impaired ability to discriminate response-produced feedback.

In the study by Van Hoesen et al (1972), an analysis of run lengths was made, and it was found that rats with septal lesions had shorter and less variable run lengths than controls on both FR-6 and FR-8 schedules on the ratio lever. On the latter schedule, the shorter run lengths led to more efficient performance on the part of operated rats (early responses did not reset the ratio counter in this study). It was also reported that an 8-day "retention" interval produced a greater tendency toward shorter run lengths in the operated rats. Contrary to the conclusions of Ellen and Kelnhofer (1971), Van Hoesen et al (1972) concluded that septal lesions did not

* Supported by grants from the National Institutes of Mental Health, MH-24612 to the first author and MH 24722 to the second author, and by grants from the Rutgers Research Council to the first and second authors.

tSend reprint requests to Charles Flaherty, Rutgers University, Psychology Department, 88 College Ave., New Brunswick, New Jersey 08903.

$\uparrow+$ Now at Natural Sciences Collegium, Eckerd College, St. Petersburg, Florida. produce a deficiency in the discrimination of response-produced feedback, and the lesions may even have led to "an increase in sensitivity to the feedback associated with emitted behavior [1972, p. 121, italics added]."

In each of the studies noted above, the authors chose not to explain the behavioral changes in terms of the more traditionally invoked notion of inhibitory deficits. Such explanations could, however, provide a number of predictions concerning the behavior of rats with septal lesions in this task. In view of the directly opposing conclusions based on an explanation in terms of response feedback, alternative explanations such as inhibition deficits should not be discounted until additional information is available.

The present experiment was conducted to obtain more information on the effects of septal lesions on the ratio "counting" task. This experiment differed from earlier studies in that: (a) a discrete-trial leverpress task was used rather than a free operant, (b) reward-lever response frequency was recorded as a function of response position on the ratio lever, (c) effects of a retention interval unconfounded by possible state-dependent effects was examined, and (d) the rats were shifted from a small to a large reward as a possible test of a motivational hypothesis of lesion effects.

\section{METHOD}

\section{Subjects}

Eleven female Sprague-Dawley strain rats obtained from Carworth Labs were used as subjects. The rats were housed individually under constant illumination and maintained at $80 \%$ of their free-feeding weight.

\section{Surgery and Histology}

Following anesthesia with Equithesin $(.25 \mathrm{cc} / 100 \mathrm{~g}$ body weight), bilateral septal lesions were produced in six rats by passing $1.5 \mathrm{~mA}$ of anodal current for $20 \mathrm{sec}$ through a stainless steel electrode lowered stereotaxically to the DeGroot coordinates 7.6 A-P, $1.0 \mathrm{H}$, and 0.5 on either side of the midline.

Following behavioral testing, the operated rats were injected with an overdose of Equithesin and perfused intracardially with saline followed by formal saline. The brains were removed and stored in a glucose-formalin solution until histological examination. The histological verification of the lesion site was 


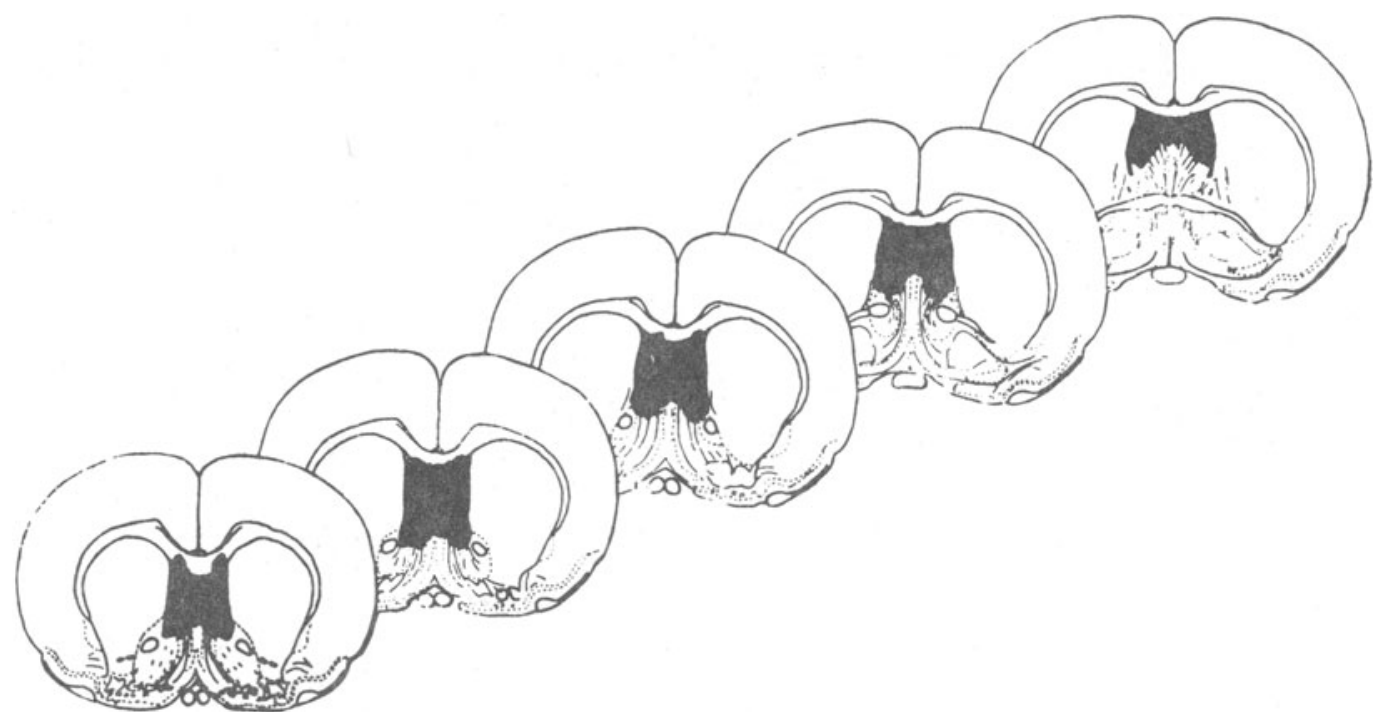

Fig. 1. Composite coronal reconstructions from the König-Klippel atlas of the maximal septal damage in the anteriorposterior plane.

accomplished by staining 60 -micron frozen brain slices with Luxol blue and examining the stained tissue microscopically.

\begin{abstract}
Apparatus
Testing was conducted in three identical Plexiglas chambers, $24 \mathrm{~cm}$ on each side and $20 \mathrm{~cm}$ high. The chambers were housed in sound-attenuating wooden boxes, equipped with two retractable levers located $6.5 \mathrm{~cm}$ from the floor, $12 \mathrm{~cm}$ apart, and extending $2.5 \mathrm{~cm}$ into the chamber. A recessed foodcup was centrally located between the levers. Chamber events were programmed and responses recorded via electromechanical relay equipment located in an adjacent room.
\end{abstract}

\section{Procedure}

There were 5 pretraining days. On Day 1, 3 weeks following surgery, all rats were given magazine training which consisted of 50.045 -g Noyes pellets delivered on a VI $30-\mathrm{sec}$ schedule. Both levers were retracted from the chamber during magazine training. On Day 2, the rats were left in the chamber until they had made 20 crf presses on the right lever, the lever that would be the reward lever during training. The left lever was not inserted into the chamber on Day 2. On Day 3, both levers were inserted and the rats were required to make at least one press on the left lever and then one on the right lever to obtain reinforcement. When a left-right sequence was completed, a single pellet was delivered and both levers retracted for a VI 30-sec intertrial interval (ITI). There were 10 such trials on Day 3. On Days 4 and 5 of pretraining, the response requirement on the left lever was three presses. When the sequence of three on the left and one on the right was completed, reinforcement was delivered and both levers retracted for the ITI. Ten trials were given on both Days 4 and 5. On the next day and throughout the remainder of the experiment, the response requirement was five presses on the left lever and one on the right. Response measures recorded included the total number of left-lever (ratio lever) and right-lever (reward lever) presses, as well as the number of reward-lever presses made on each trial before any ratio-lever responses were made, and after one, two, three, and four ratio-lever responses were made.

The experiment was conducted in three phases. In the first (acquisition) phase, the rats were run for 10 consecutive days with a single 45-mg Noyes pellet as the reward. In the second (retention) phase, there was a 4-day break in running, followed by 2 more days with the single-pellet reward. In the third (large reward) phase, all rats were shifted to a five-pellet reward. Testing was continued with the large reward for 5 days. In all phases, there were 10 trials per day with a VI 45 -sec ITI.

\section{RESULTS}

\section{Histology}

The lesions involved nearly total destruction of septal tissue. The damage extended dorsally to the corpus callosum, ventrally to the anterior commissure, posteriorly to the descending columns of the fornix, and anteriorly to the medial parolfactory area. Damage extended laterally to the walls of the lateral ventricles. One animal had some sparing of the medial septum, but this was not correlated with any obvious behavioral differences. A reconstruction of the typical lesion is shown in Fig. 1.

\section{Acquisition}

The total ratio- and reward-leverpresses on each day of testing are presented in Fig. 2. In interpreting these data, it would be helpful to remember that optimal responding would require 50 presses on the ratio lever and 10 presses on the reward lever each day. Early in the acquisition phase, both lesioned and control animals responded considerably more on the reward than on the ratio lever. Over the 10 days of acquisition training, the control rats demonstrated a steady decline in reward-lever responses and, at the end of 10 days, they were responding less on the reward lever than on the ratio lever. The operated rats showed no evidence of a systematic decline in reward-lever responses across the acquisition phase. The operated rats did, however, tend to respond somewhat less on the ratio lever, coming 


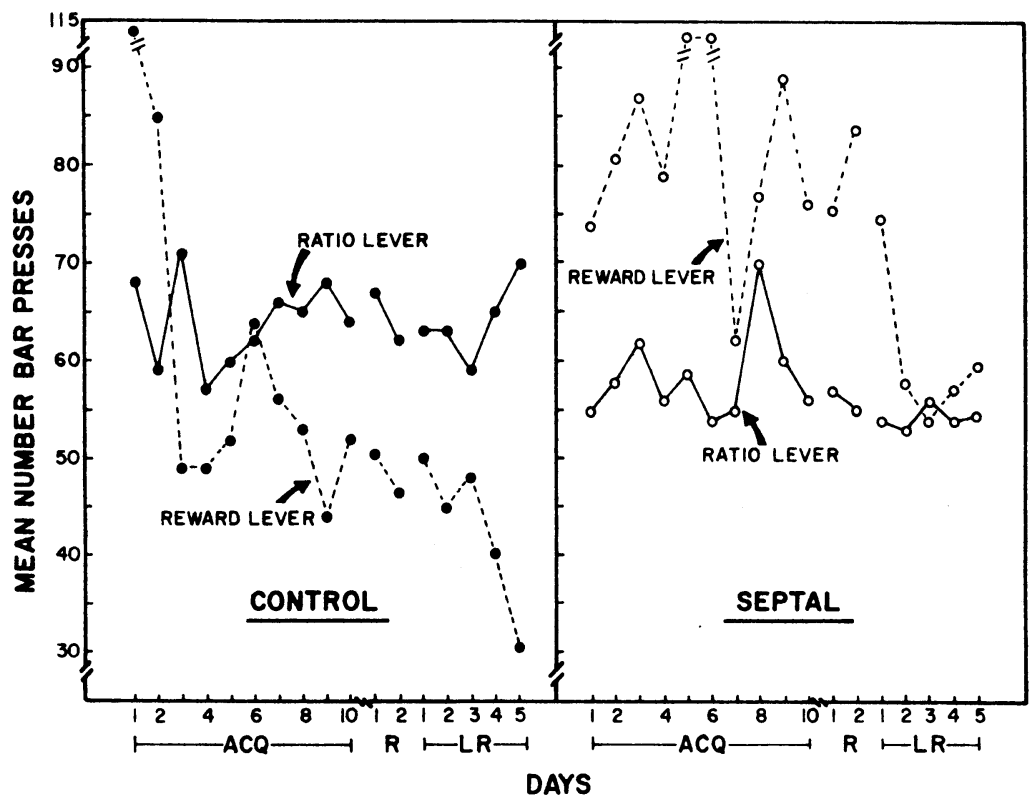

Fig. 2. Mean number of presses on ratio and reward levers as a function of experimental conditions: ACQ $=10$ days with one-pellet reward; $R=2$ days with one-pellet reward following a 4-day retention interval; $L R=5$ days with a five-pellet reward.

closer than the controls to the minimum of 50 responses per session.

Statistical support for these observations was provided by an analysis of variance performed on the Day 1 and Day 10 data. The analysis indicated a reliable Lesion by Lever by Day interaction $(\mathrm{F}=5.64, \mathrm{df}=1 / 9, \mathrm{p}<.05)$. Subsequent analysis of the interaction data with Fisher's LSD procedure $(p=.05$, two-tail) showed that both groups responded more to the reward lever on Day 1; this difference was still present in the operated animals on Day 10, but the controls now responded reliably less on the reward than on the ratio lever. The difference between operated and control animals on ratio-lever responses was not reliable. A further analysis of the terminal acquisition data (Days $9 \& 10$ combined) is shown in the first row of Fig. 3. This figure shows the median number of reward-lever responses for both groups as a function of position within the FR-5 schedule on the ratio lever. It can be seen that the operated animals were more likely than the controls to respond on the reward lever at every position on the ratio schedule. These differences were statistically reliable by a Mann-Whitney test after two $(p<.009)$ and after four $(p=.02)$ ratio-lever responses.

\section{Retention Test}

It is apparent from both Figs. 2 and 3 that the passage of a 4-day retention interval had no systematic effect on the performance of either lesioned or control animals. During this 2-day retention test, the operated animals continued to respond reliably more than the controls on the reward lever while responding at approximately the same rate on the ratio lever (Lesion by Lever interaction $\mathrm{F}=5.41, \mathrm{df}=1 / 9, \mathrm{p}<.05)$.

\section{Large Reward}

The shift to a five-pellet reward led to a decline in reward-leverpresses in both groups (Fig. 2). In the control rats, this decline accentuated the already existing difference between reward and ratio-lever responding whereas, in the operated rats, the decline was sufficient to bring reward-lever responding down to the same lever as ratio-lever responding. Statistical analysis indicated that, for the operated rats, there was no reliable response-rate difference between the ratio- and reward-levers $(\mathrm{F}=2.14, \mathrm{df}=1 / 45, \mathrm{p}>.05)$. However, in the case of the control rats, responding on the reward lever was reliably less than responding on the ratio lever $(F=52.17, d f=1 / 36, p<.01)$, and became increasingly so over the course of the large reward phase (Interaction $F=2.90, d f=4 / 36, p<.05)$.

A more detailed examination of the effects of the shift to large reward is presented in Fig. 3. It is apparent that the reduction in reward-lever responding that occurred was principally in the earlier stages of the ratio run. However, the operated rats still tended to switch over to the reward lever more than the controls as the ratio run neared its completion. This greater anticipatory responding in the operated rats was reflected in reliable linear trends (Ferguson, 1971, p. $337 \mathrm{ff}$ ) across the ratio positions on large reward Days $1(\mathrm{p}<.01), 2(\mathrm{p}<.01)$, $3(p<.02)$ and $5(p<.001)$. The control rats showed no reliable linear trends on any test day.

\section{DISCUSSION}

The principal result of this experiment was that rats with septal lesions are more likely than control rats to shift to the reward lever prior to completion of 


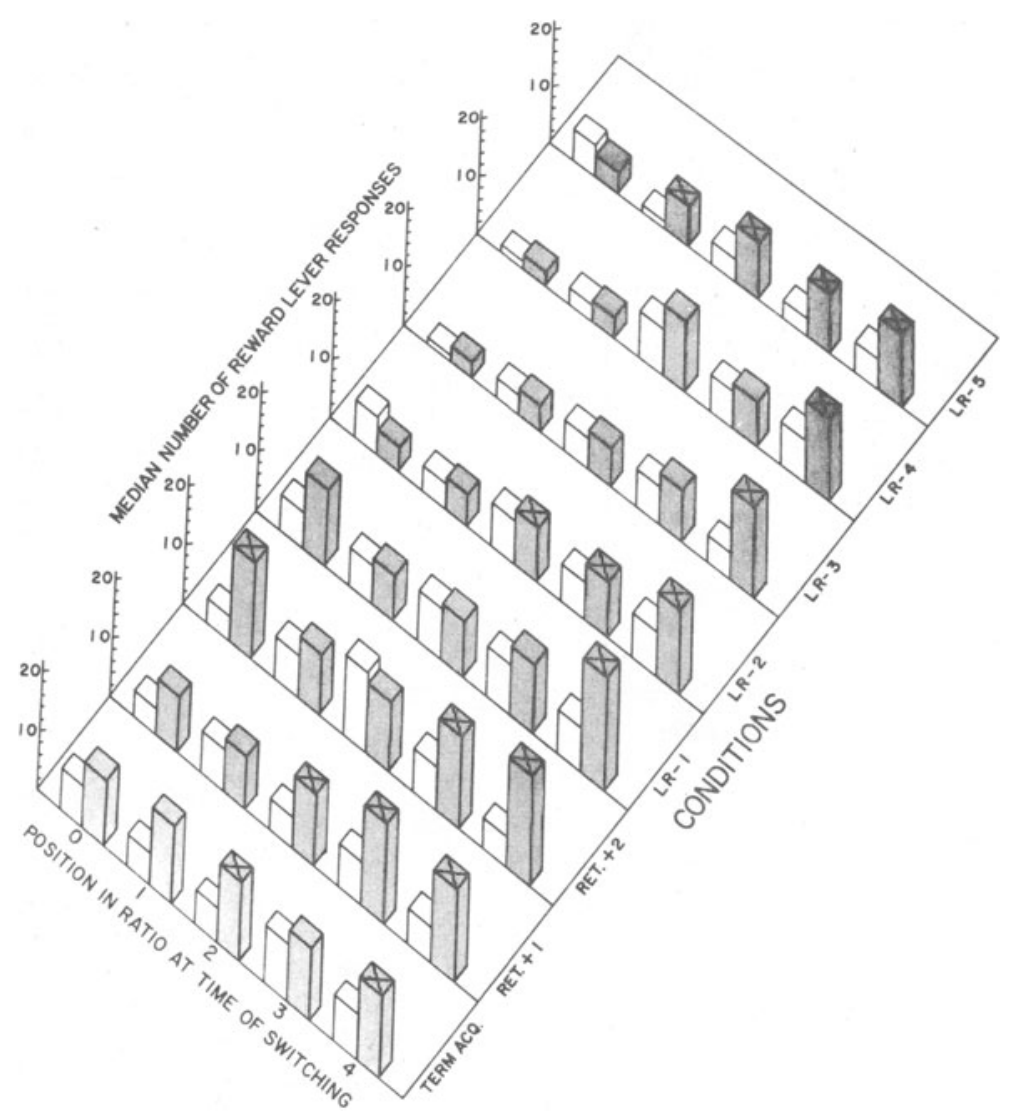

Fig. 3. Median number of responses on reward lever as a function of number of responses made on the ratio lever. The data presented include terminal acquisition as well as each retention test day and each day on the large reward. Shaded bars represent the operated rats and a shaded bar with an $X$ on top indicates a statistically reliable difference (Mann-Whitney, $p<.05$ between operated and control rats. responding on the ratio lever. Although a satisfactory theoretical description of these results remains elusive, the results of our experiment provide some additional insight into the nature of the behavioral change.

Earlier studies obtaining similar results have attributed the altered performance of the operated rats to a change in the sensitivity to response-produced feedback. One of these studies attributed the change to increased sensitivity (Van Hoesen et al, 1972) whereas the other study attributed the change to decreased sensitivity (Ellen \& Kelnhofer, 1971). Clearly, the exact relationship between the performance of operated and control animals will depend on the demand characteristics of the experiment. For example, in the study in which a presumed decrease in the sensitivity to response feedback was found to be an adequate explanation of the data, the procedures used were such that anticipatory responses on the reward lever reset the ratio counter. Thus, operated rats, making more anticipatory responses, would also have an opportunity to respond more on the ratio lever; which they did. Given these results of greater responding by the operated rats on both levers, the decreased sensitivity interpretation is not unreasonable. In the Van Hoesen et al (1972) experiments, shifts in the response requirements on the ratio lever (e.g., FR-5 to FR-8) were included as part of the design. The results showed that operated rats adjusted to or tracked such shifts better than control rats. Given these data, the interpretation of increased sensitivity to response feedback is not unreasonable. However, it seems quite likely that the more rapid adjustment of the operated rats to the schedule was due to the more frequent sampling of the reward lever that occurred in these rats and not necessarily due to any increased sensitivity to response-produced feedback.

A possible alternative explanation for the greater anticipatory responding in septal rats is in terms of a motivational dysfunction. It has been demonstrated repeatedly that rats with septal lesions overrespond to motivationally relevant stimuli such as sucrose and quinine solutions (Beatty \& Schwartzbaum, 1967, 1968). Given these data, it is not unreasonable to interpret the anticipatory responding in terms of enhanced incentive motivation. Ellen and Kelnhofer (1971) attempted to investigate the motivational interpretation by prefeeding their subjects before alternate daily sessions. The results indicated that prefeeding did not decrease the number of anticipatory responses in either lesioned or control animals, and, in fact, if any trend was evident, it was toward an increased number of anticipatory responses on preload days. However, prefeeding or stomach loads may not be the best way to manipulate motivational levels in septal rats 
since there is some evidence that rats with septal lesions are less sensitive than controls to postingestive effects (e.g., Singh \& Meyer, 1968; Flaherty \& Hamilton, 1971). In the present study, we attempted to investigate potential motivational effects by increasing the amount of reward received for barpressing. Previous studies have indicated that a within-subjects difference of one pellet vs five pellets is sufficient to produce apparent enhanced motivation in normal rats under the five-pellet conditions (e.g., Davenport \& Flaherty, 1969). If the differences in anticipatory responding were due to enhanced incentive motivation in the rats with septal lesions, it might be expected that the degree of anticipatory responding would be raised in both groups by increasing the amount of reward. This was clearly not the case. Responding on the reward lever declined abruptly subsequent to the shift to large reward, although operated animals still responded more than controls and showed a clear trend toward increased probability of responding as the ratio reared completion. Thus, between the present study and the Ellen and Kelnhofer (1971) experiment, there is little evidence that a motivational interpretation will provide an understanding of anticipatory responding in the two-lever paradigm.

Still another possible interpretation, one that has frequently been applied to the effects of septal lesions, is that of a deficiency in response inhibition (McCleary, 1966). However, such an interpretation is not always appropriate for septal-lesion effects (e.g., Flaherty \& Hamilton, 1971; Kelsey \& Grossman, 1971) and it is not clear how this interpretation could make any precise prediction as to what should happen in the two-lever task. For example, should the deficit in inhibition apply to the ratio lever? If so, operated rats should make more total responses on the ratio lever, but the data show quite clearly that there is little difference in ratio responding. The small difference that does exist is in the direction of the operated rats responding less on the ratio lever. Should the deficit in inhibition apply to the reward lever? Here, the operated rats do make more responses than the controls, but the pattern of the excess responses is not clearly predicted by a simple inhibitory-deficit interpretation. One could say that the primary basis for the deficit lies in an impaired ability to inhibit switching from the ratio lever to the reward lever until the ratio is completed. However, this interpretation is not behaviorally different from the failure-to-discriminate response-feedback interpretation and neither interpretation really goes much beyond the data.

Furthermore, Kelsey and Grossman (1971) found that rats with septal lesions tended to make anticipatory errors rather than perseverative errors in a situation which was designed to discriminate between these two types of deficits. In the present experiment, the use of retracting levers provided a clear termination of each discrete trial and prevented the occurrence of perseverative errors. The reappearance of the levers for the subsequent trial provided a clear onset cue for the required behavioral sequence and it is likely that any early response on the reward lever represented anticipatory rather than perseverative responses. The clear linear gradient obtained, particularly under large reward conditions, supports the anticipatory rather than the perseverative interpretation of the reward-lever responses.

Thus, at the present time, we are left with the basic result that rats with septal lesions tend to make more anticipatory responses on the reward lever, and with no adequate explanation in terms of presumed psychological processes underlying the behavior.

One other aspect of these data deserves some comment. Van Hoesen et al (1972) found that an 8-day retention interval was sufficient to alter the performance of operated rats on this task. In the present study, there was no effect of a 4-day retention interval. However, in the Van Hoesen study, the surgery was conducted during the "retention" interval whereas in the present study the surgery was conducted well before the retention interval. Thus, the "retention' intervals used in the two studies were not of the same type. In addition, the 4-day retention period used in the present experiment has been found in the past to be sufficient for producing large differences between operated and control rats in another task (Flaherty, Capobianco, \& Hamilton, 1973).

\section{REFERENCE}

Beatty, W. W., \& Schwartzbaum, J. S. Enhanced reactivity to quinine and saccharin solutions following septal lesions in the rat. Psychonomic Science, 1967, 8, 483-484.

Beatty, W. W., \& Schwartzbaum, J. S. Consummatory behavior for sucrose following septal lesions in the rat. Journal of Comparative \& Physiological Psychology, 1968, 65, 93-102.

Buckland, G. H., \& Schwartzbaum, J. S. Fixed-ratio performance for sucrose under nondeprived conditions following septal lesions in rats. Psychonomic Science, 1970, 18, 271-273.

Davenp ort, J. W., \& Flaherty, C. F. Extinction of differential reward magnitude discrimination in a discrete bar-pressing situation. Psychonomic Science, 1969, 14, 29-30.

Ellen, P., \& Kelnhofer, M. Discrimination of response feedback following septal lesions. Psychonomic Science, 1971, 23, 94-96.

Ellen, P., \& Powell, E. W. Effects of septal lesions on behavior generated by positive reinforcement. Experimental Neurology, 1962, 6, 1-11.

Ellten, P., Wilson, A. S., \& Powell, E. W. Septal inhibition and timing behavior in the rat. Experimental Neurology, 1964, 10, $120-132$.

Ferguson, G. A. Statistical analysis in psychology \& education. New York: McGraw-Hill, 1971.

Flaherty, C. F., Capobianco, S., \& Hamilton, L. W. Effect of septal lesions on retention of negative contrast. Physiology \& Behavior, 1973, 11, 625-631.

Flaherty, C. F., \& Hamilton, L. W. Responsivity to decreasing sucrose concentrations following septal lesions in the rat. Physiology \& Behavior, 1971, 6, 431-437. 
Kelsey, J. E., \& Grossman, S. P. Nonperseverative disruption of behavioral inhibition following septal lesions in rats. Journal of Comparative \& Physiological Psychology, 1971, Vol. 75, 2, 302-311.

MacDougall, J. M. Van Hoesen, G. W., \& Mitchell, J. C. Anatomical organization of septal projections in maintenance of DRL behavior in rats. Journal of Comparative \& Physiological Psychology, 1969, 68, 568-575.

Mechner, F. Probability relations within response sequences under ratio reinforcement. Journal of the Experimental Analy sis of Behavior, 1958a, 1, 109-121.

Mechner, F. Sequential dependencies of the length of consecutive response runs. Journal of the Experimental Analysis of Behavior, $1958 \mathrm{~b}, 1,229-233$.

McCleary, R. A. Response-modulating functions of the limbic system: Initiation and suppression. In: E. Stellar and J. M. Sprague (Eds.), Progress in physiological psychology. Vol. 1. New York: Academic Press, 1966.
Singh, D, \& Meyer, D, R. Eating and drinking by rats with lesions of the septum and the ventromedial hypothalamus. Journal of Comparative \& Physiological Psychology, 1968, 65, 163-166.

Van Hoesen, G. W. MacDougall, J. M. \& Mitchell, J. C. Discrimination of emitted behavior following septal area lesions in rats. Journal of Comparative \& Physiological Psychology, 1972, 80, 106-122.

(R eceived for publication June 18, 1974; revision received July 30,1974 .) 